\title{
EEG-based research on brain functional networks in cognition
}

\author{
Niannian Wang, Li Zhang and Guozhong Liu* \\ School of Instrument Science and Opto-Electronics Engineering, Beijing Information Science and \\ Technology University, 12 East Qing he xiao ying RD, Hai dian Dist, Beijing 100192, China
}

\begin{abstract}
Recently, exploring the cognitive functions of the brain by establishing a network model to understand the working mechanism of the brain has become a popular research topic in the field of neuroscience. In this study, electroencephalography (EEG) was used to collect data from subjects given four different mathematical cognitive tasks: recite numbers clockwise and counter-clockwise, and letters clockwise and counter-clockwise to build a complex brain function network (BFN). By studying the connectivity features and parameters of those brain functional networks, it was found that the average clustering coefficient is much larger than its corresponding random network and the average shortest path length is similar to the corresponding random networks, which clearly shows the characteristics of the small-world network. The brain regions stimulated during the experiment are consistent with traditional cognitive science regarding learning, memory, comprehension, and other rational judgment results. The new method of complex networking involves studying the mathematical cognitive process of reciting, providing an effective research foundation for exploring the relationship between brain cognition and human learning skills and memory. This could help detect memory deficits early in young and mentally handicapped children, and help scientists understand the causes of cognitive brain disorders.
\end{abstract}

Keywords: EEG, brain function network, small-word property, cognitive memory

\section{Introduction}

Neural connections in the brain can be divided into structural brain networks, functional brain networks, and effective brain networks [1]. These networks have small-world attributes, and studying complex brain networks has become an important direction for EEG research [2,3].The structure and function of the human brain is extremely complex, requiring neurons, clusters of neurons, or multiple brain regions to achieve the differentiation and integration of brain functions through mutual cooperation. Differentiation and integration are the two organization principles of the human brain. Additionally, completing a simple task requires complex brain coordination among different functional areas, although different regions correspond to different functional particularities $[4,5]$. Analyzing mutual linkages and effects among different brain regions performing different cognitive tasks is currently a focus in brain research.

\footnotetext{
*Address for correspondence: Guozhong Liu, School of Instrument Science and Opto-Electronics Engineering, Beijing Information Science and Technology University, 12 East Qing he xiao ying RD, Hai dian Dist, Beijing 100192, China. Tel.: 0086 13801072335; Fax: 0086-10-82426915; E-mail: liuguozhong@bistu.edu.cn.
} 


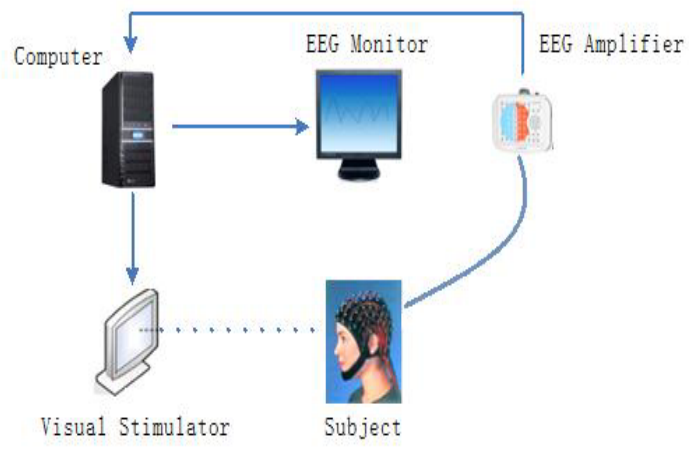

Fig. 1. Experimental system diagram.

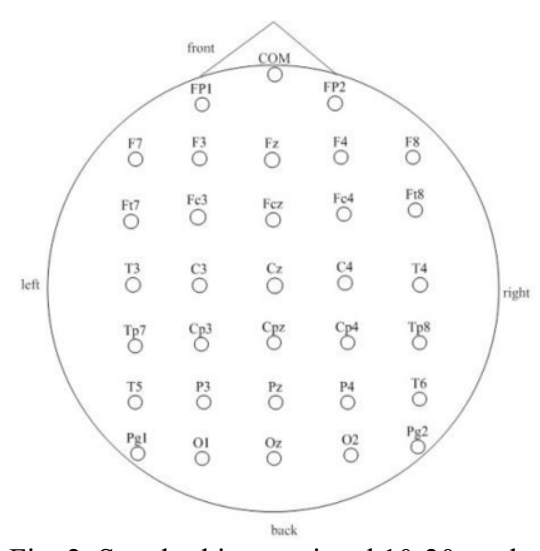

Fig. 2. Standard international 10-20 method.

Several studies focus on the construction of the resting-state brain function network, which is the functional connectivity in the brain at a resting state [6]. However, the topological property of the brain network is associated with cognitive behavior. The brain network changes during cognitive processing or while conducting activities, and shows different patterns for different cognitive tasks; thus, the building and analysis of brain functions when performing tasks is a popular topic in brain research $[7,8]$. This study is based on the pattern analysis of different cognitive tasks. The major task is the sampling of EEG signals from normal subjects subjected to different visual stimuli, followed by filtering, extracting features, and building and analyzing networks. Studying the structure and dynamic characteristics of the brain network is expected to provide new information regarding the mechanism of brain disease and the evaluation of brain memory and learning defects in some mentally disadvantaged children, and to aid in developing clinical diagnosis methods for encephalopathy.

\section{Experimental system and scheme design}

\subsection{Experimental system design}

The experimental system includes a subject, a computer, a visual stimulator, an EEG amplifier, and a real-time EEG monitor (see Figure 1). The subject provides cognitive responses to stimulation signals based on the VC program. The EEG signals are amplified by the EEG amplifier, displayed on the monitor, and recorded and processed by the computer. An EEG amplifier with 32 channels was adopted to record signals. The recording electrodes were placed according to the international standard method: the 10-20 arrangement (see Figure 2).

\subsection{Experimental scheme design}

The subject wore an electrode cap and sat on a chair, with eyes forward to a stimulator display screen 1.1 meters in front of the subject. The subject's shoulders and arms were in a naturally relaxed position. The subject was asked to maintain silence. Eight undergraduate students were engaged in the experiment, with each experimental acquisition lasting for approximately half a minute. Resting states lasted 4 seconds, whereas the task state lasted 9 seconds; the sequence diagram is shown below (see Figure 3). 
This stimulus package includes four types of mathematical cognitive tasks: clockwise numbers, counter-clockwise numbers, clockwise letters, and counter-clockwise letters. Participants were asked to recite what they saw in different cognitive tasks.

\section{Date processing}

The data processing flow chart is presented below (see Figure 4).

\subsection{Date preprocessing}

The EEG signal contains a relatively wide frequency range from $0.1-100 \mathrm{~Hz}$, which is weak and is accompanied by other noises - non-EEG artifacts such as eye movement and blinking [9]. In our study, we selected frequencies ranging from $8-30 \mathrm{~Hz}$ using the Chebyshev band-pass filtering method. Figure 5 presents the waves of the EEG lead T5 in the more active posterior temporal region before and after filtering.

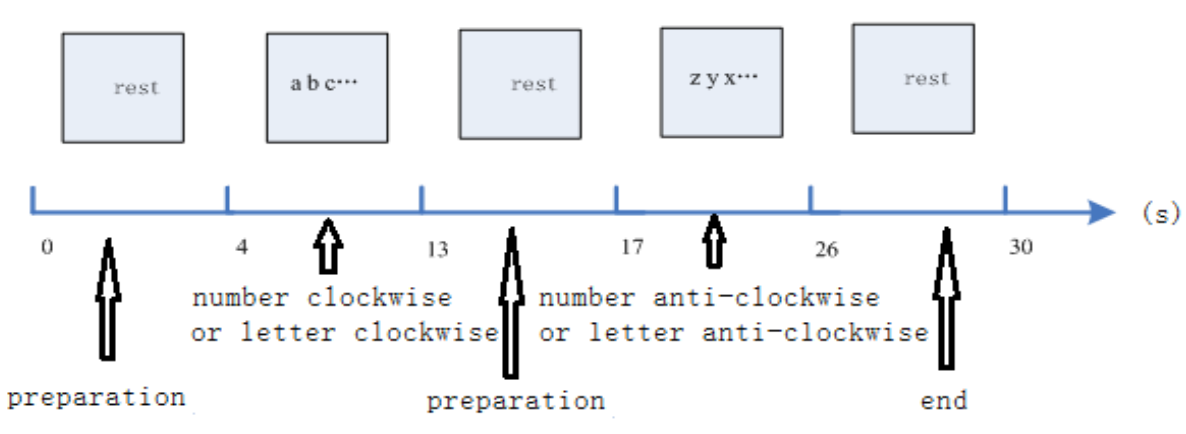

Fig. 3. Experimental stimulation sequence diagram.

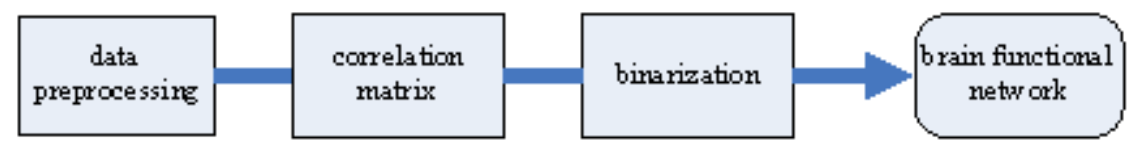

Fig. 4. Brain function network construction flow chart.

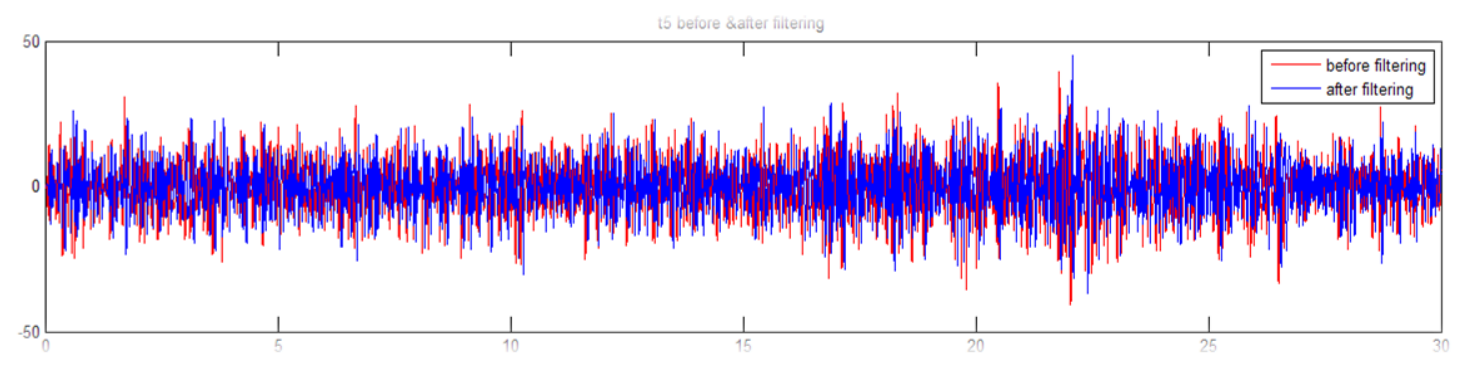

Fig. 5. Waves of T5 before and after filtering. 


\subsection{Frequency-domain correlation analysis}

The correlation co-efficiency between two leads with a distance of $15 \mathrm{~cm}$ can be as high as 60 to $70 \%$ [10]; thus, the frequency-domain correlation method based on FFT is used for correlation analysis to explore the link between brain areas. Figure 6 depicts the correlation between 16 typical leads, which are evenly placed on the head in accordance with the international 10-20 standard method. Therefore, the following data processing is based on the data set of those 16 typical leads.

\subsection{The construction of the brain function network}

The construction of the brain network is primarily based on the graph theory, which represents the brain as a map. The area covered by the EEG electrode corresponds to a node and the connection between the nodes represents their relationship. The correlation analysis method is used to analyze the band-pass filtered signals to obtain symmetric correlation matrixes, and each element of the matrix represents the correlation between nodes $i$ and $j$. To reduce the effect between weak correlation leads, the binarization process is necessary after obtaining the correlation matrix. This paper uses the between-cluster variance method to select the appropriate threshold. When the matrix element is greater than the threshold, there is a functional connection between the two brain areas; otherwise, it does not exist. A brain network diagram (BND) was constructed, as shown in Figure 7.

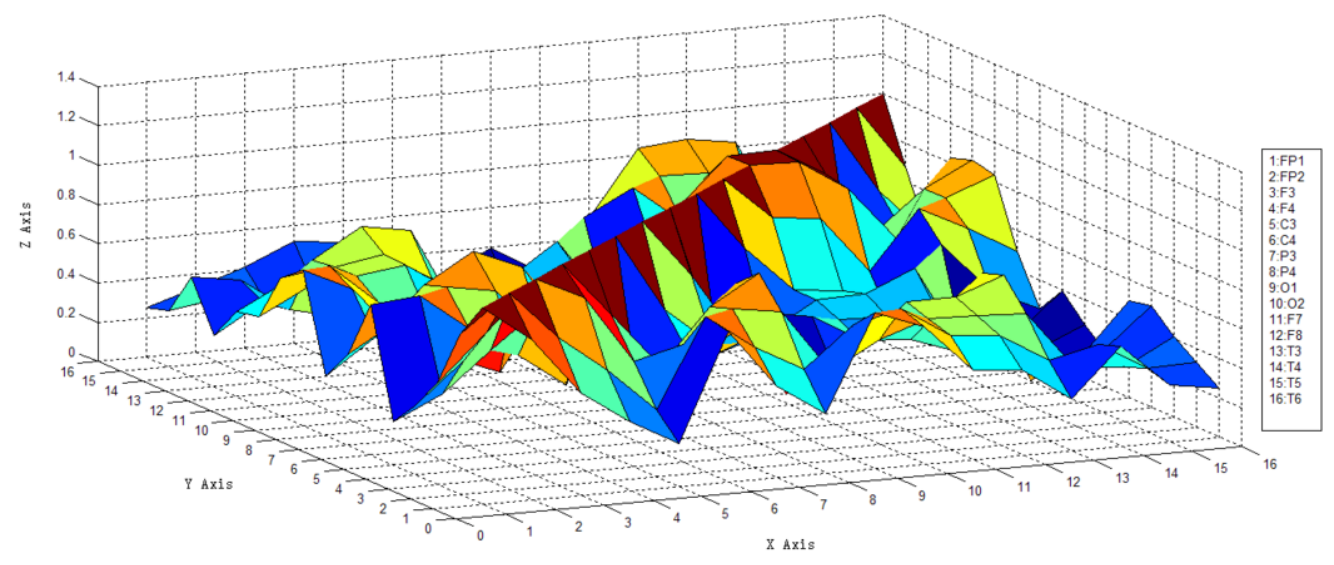

Fig. 6. The correlation between 16 typical leads (the $\mathrm{X}$ and $\mathrm{Y}$ axes are the 16 leads, the $\mathrm{Z}$ axis is the correlation value.).
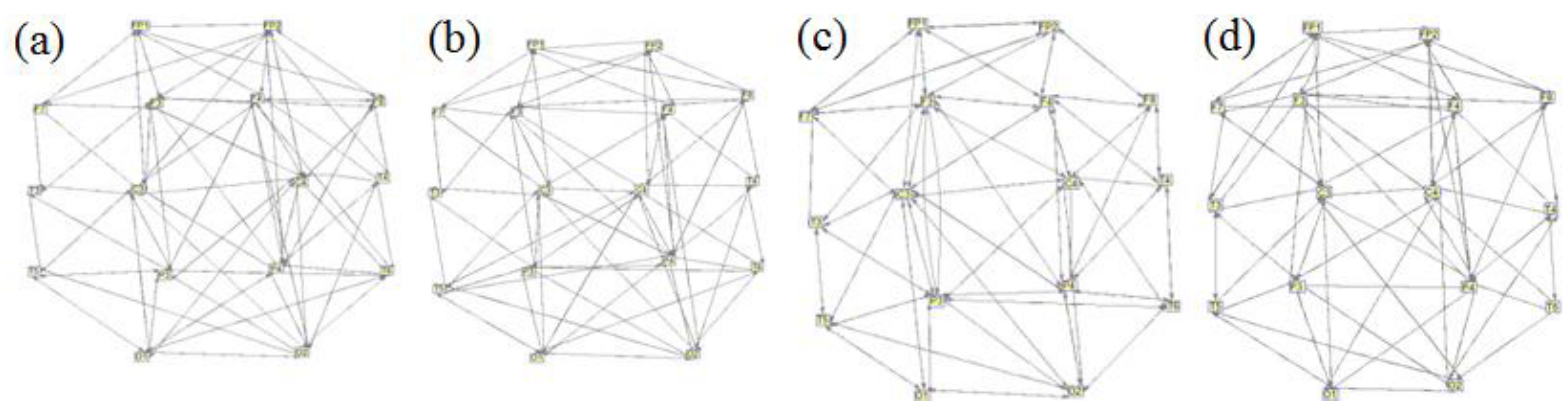

Fig. 7. Brain functional networks in four tasks, counting: (a) clockwise numbers, (b) anti-clockwise numbers, (c) clockwise letters, (d) anti-clockwise letters. 


\section{Network parameter analysis}

The following network parameters are analyzed to verify the features of human brain functions.

- Node degree: Each region of the EEG lead measured is defined as a node of the BFN. The node degree is defined as the number of vertices connected with the node.

- Shortest path length: A path of the minimum number of edges between two network nodes $i$ and $\mathrm{j}$ is the shortest path to this network, and the number of edges through the path between $\mathrm{i}$ and $\mathrm{j}$ is the shortest path length $\mathrm{l}_{\mathrm{i}, \mathrm{j}}$. The shortest path length $\mathrm{L}$ is the average shortest path length between any two nodes in the network.

- Clustering coefficient: The clustering coefficient of node $i$ is the ratio between the actual number of edges $e_{i}$ and the highest number of possible connected edges with node $i$, that is, $C_{i}=\frac{2 e_{i}}{k_{i}\left(k_{i}-1\right)}$. The average clustering coefficient refers to the average value of all nodes.

- Small-world properties: Studies have shown that the brain network is a complex network system between the regular network and the random network, and "small-world" is an important statistical property of general complex networks [11], and the clustering coefficient and the average shortest path length are two important parameters to characterize network properties. The regular network has a higher cluster coefficient and a longer shortest path length, whereas the random network presents the opposite. These two indicators can be quantified by the "smallworld" network properties, that is:

$$
\begin{aligned}
& \gamma=\mathrm{C}_{\text {real }} / \mathrm{C}_{\text {random }}>1 \\
& \lambda=\mathrm{L}_{\text {real }} / \mathrm{L}_{\text {random }} \sim 1
\end{aligned}
$$

The subscript "random" represents a random network, and the subscript "real" represents a real network. Later, Humphries, who unified the two metrics for a scalar $\sigma$, determined:

$$
\sigma=\gamma / \lambda
$$

When $\sigma>1$, the network has a "small-world" property. The larger the $\sigma$, the stronger the "smallworld" network property.

Figure 8 presents the value of the clustering coefficient for one of the subjects in different cognitive tasks. As presented in the figure, the memory cognitive stimulation using the numbers and letters will stimulate the frontal pole, temporal, occipital, and parietal regions. Among them, the activity in the frontal pole and the temporal regions are more obvious.

(a)

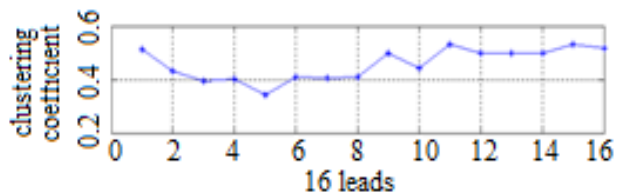

(b)

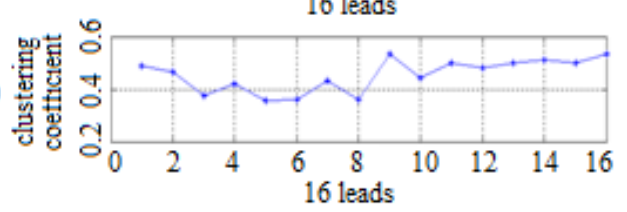

(c)
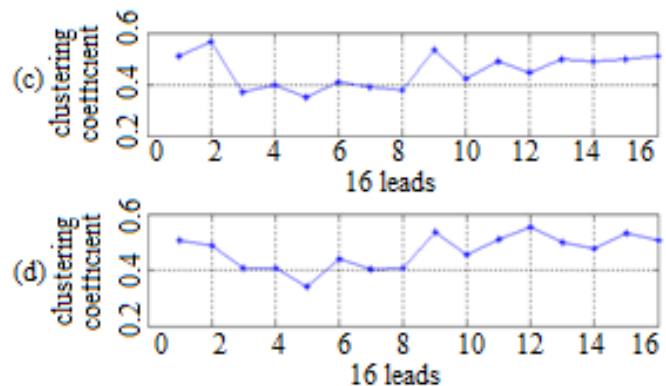

Fig. 8. Clustering coefficient of (a) clockwise numbers, (b) counter-clockwise numbers, (c) clockwise letters, and (d) counterclockwise letters. 


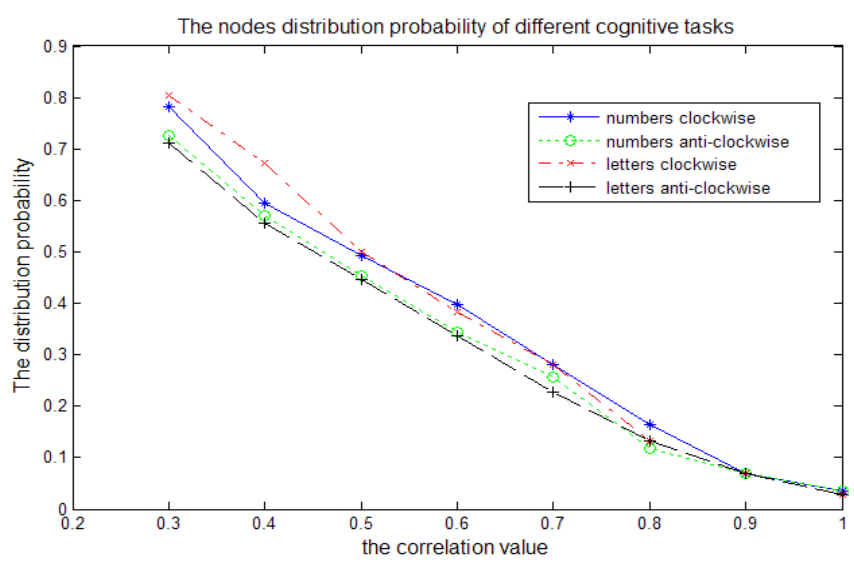

Fig. 9. The nodes distribution probability of different cognitive tasks.

Figure 9 shows the nodes' distribution probability under different cognitive tasks. The results for counter-clockwise letters decline at the slowest rate.

Table 1 presents the network parameters of the 8 subjects (M1, M2, M3, M4, M5, M6, M7, M8), including the number of network connection edges, the average clustering coefficient $\mathrm{C}$, the average degree value $\mathrm{K}$, the average shortest path length $\mathrm{L}$, and the parameter $\sigma$.

Analysis of the above table and figures draws the following conclusions:

- Denser network nodes are primarily distributed in the frontal, temporal, and occipital parietal areas, which is consistent with the cognitive theory: visual stimulation-activated brain regions are closely related to attention, thinking, understanding, and other cognitive tasks.

- Under the four types of cognitive tasks, the maximum connection number for the BFN is for the task involving counter-clockwise letters. This indicates that in more complex tasks, the contacts between various brain regions show a higher number of close relationships, making the network more resistant to outside interference.

- Core network nodes exist, which have higher degree values with many connections. They play important roles in the network.

- The average shortest path length value under the counter-clockwise letter task tends to be the lowest. The results indicate that the speed of the brain when dealing with information is faster during relatively complex memory cognitive task, and that the transmission and exchange of information between the nodes are more efficient.

- The average clustering coefficient for the BFN in the four types of cognitive tasks is higher than that for the random network with the same scale, whereas the average shortest path length is not markedly different for the BFN and the random network. The four types of task networks present small-world properties, and complex memory cognitive networks (counter-clockwise letters) have the largest average clustering coefficient and the minimal average shortest path length, which show the most obvious characteristics of small-world networks.

\section{Conclusions and discussions}

This paper studies the brain functional networks constructed by EEG data for four visual stimuli cognitive tasks and analyzes BFN property parameters, validating that the four types of BFNs con- 
Table 1

Parameter analysis of the BFN for four different cognitive tasks

\begin{tabular}{|c|c|c|c|c|c|c|c|}
\hline parameter & & nodes & connection & $\mathrm{C}$ & $\mathrm{K}$ & $\mathrm{L}$ & $\bar{\sigma}$ \\
\hline \multirow[t]{8}{*}{ clockwise numbers } & M1 & 16 & 66 & 0.44965 & 8.875 & 1.500 & 1.35 \\
\hline & M2 & 16 & 63 & 0.45855 & 9.250 & 1.475 & 1.40 \\
\hline & M3 & 16 & 52 & 0.45358 & 7.500 & 1.6833 & 1.22 \\
\hline & M4 & 16 & 57 & 0.46306 & 8.125 & 1.6167 & 1.30 \\
\hline & M5 & 16 & 56 & 0.46125 & 8.000 & 1.6583 & 1.25 \\
\hline & M6 & 16 & 52 & 0.49670 & 7.500 & 1.7583 & 1.27 \\
\hline & M7 & 16 & 54 & 0.50994 & 7.750 & 1.7417 & 1.32 \\
\hline & M8 & 16 & 54 & 0.49872 & 7.750 & 1.6583 & 1.36 \\
\hline \multirow{2}{*}{$\begin{array}{l}\text { Ave. } \\
\text { standard deviation }\end{array}$} & & 16 & 56 & 0.47393 & 8.09375 & 1.63645 & 1.31 \\
\hline & & & 4.81534 & 0.02222 & 0.64236 & 0.09627 & 0.05667 \\
\hline \multirow{8}{*}{$\begin{array}{l}\text { anti- } \\
\text { clockwise numbers }\end{array}$} & M1 & 16 & 64 & 0.45998 & 8.750 & 1.525 & 1.36 \\
\hline & M2 & 16 & 62 & 0.45472 & 9.000 & 1.500 & 1.34 \\
\hline & M3 & 16 & 47 & 0.45420 & 6.875 & 1.8917 & 1.09 \\
\hline & M4 & 16 & 54 & 0.46184 & 7.750 & 1.6583 & 1.26 \\
\hline & M5 & 16 & 54 & 0.46109 & 7.750 & 1.6733 & 1.24 \\
\hline & M6 & 16 & 57 & 0.49289 & 8.125 & 1.6083 & 1.38 \\
\hline & M7 & 16 & 60 & 0.48451 & 8.500 & 1.5583 & 1.40 \\
\hline & M8 & 16 & 61 & 0.49038 & 8.625 & 1.6833 & 1.31 \\
\hline \multirow{2}{*}{$\begin{array}{l}\text { Ave. } \\
\text { standard deviation }\end{array}$} & & 16 & 57 & 0.46995 & 8.171875 & 1.637275 & 1.29 \\
\hline & & & 5.19465 & 0.01533 & 0.64933 & 0.11571 & 0.09417 \\
\hline \multirow[t]{8}{*}{ clockwise letters } & M1 & 16 & 58 & 0.45409 & 8.250 & 1.600 & 1.28 \\
\hline & M2 & 16 & 59 & 0.45590 & 7.625 & 1.6667 & 1.23 \\
\hline & M3 & 16 & 50 & 0.45897 & 7.250 & 1.725 & 1.23 \\
\hline & M4 & 16 & 58 & 0.46712 & 8.250 & 1.5833 & 1.33 \\
\hline & M5 & 16 & 50 & 0.46088 & 7.250 & 1.7167 & 1.21 \\
\hline & M6 & 16 & 57 & 0.51412 & 8.125 & 1.7083 & 1.36 \\
\hline & M7 & 16 & 52 & 0.49751 & 7.500 & 1.7750 & 1.26 \\
\hline & M8 & 16 & 53 & 0.49607 & 7.625 & 1.7333 & 1.29 \\
\hline \multirow{2}{*}{$\begin{array}{l}\text { Ave. } \\
\text { standard deviation }\end{array}$} & & 16 & 54 & 0.47558 & 7.734375 & 1.688538 & 1.27 \\
\hline & & & 3.53332 & 0.02109 & 0.39250 & 0.06263 & 0.04872 \\
\hline \multirow{8}{*}{$\begin{array}{l}\text { anti- } \\
\text { clockwise letters }\end{array}$} & M1 & 16 & 65 & 0.46739 & 9.875 & 1.4167 & 1.49 \\
\hline & M2 & 16 & 61 & 0.45738 & 8.625 & 1.5417 & 1.34 \\
\hline & M3 & 16 & 55 & 0.46286 & 7.500 & 1.6833 & 1.24 \\
\hline & M4 & 16 & 63 & 0.46468 & 8.875 & 1.5417 & 1.36 \\
\hline & M5 & 16 & 59 & 0.47947 & 8.375 & 1.5917 & 1.36 \\
\hline & M6 & 16 & 57 & 0.49399 & 8.125 & 1.6083 & 1.38 \\
\hline & M7 & 16 & 55 & 0.50633 & 7.500 & 1.6750 & 1.36 \\
\hline & M8 & 16 & 64 & 0.50669 & 9.000 & 1.5083 & 1.51 \\
\hline \multirow{2}{*}{$\begin{array}{l}\text { Ave. } \\
\text { standard deviation }\end{array}$} & & 16 & 59 & 0.47985 & 8.484375 & 1.570838 & 1.38 \\
\hline & & & 3.72282 & 0.01871 & 0.74592 & 0.08269 & 0.08016 \\
\hline
\end{tabular}

structed in the experiment exhibit small-world properties. In the four structure networks, the brain information transfers fastest for the memory task involving counter-clockwise letters; it has the minimal shortest path length and the fastest network transmission. Cognitive processes are closely related to word learning and memory. It is observed that the prefrontal, parietal, occipital, and temporal regions are activated by the cognitive process, which is consistent with results from cognition research. That is, the activation of the frontal and prefrontal area is closely related to human understanding, thinking and other rational discrimination. These areas overlap with the large brain 
regions associated with verbal working memory; the bilateral pillow top areas relate to the human visual system to process information from visual stimulation; the temporal areas are chiefly responsible for processing.

Our brain is a complex network, and mathematical cognition is a very complex cognitive process. This paper explores the relationship between brain cognition and human learning and memory, which provides important insights into this field. This study aids the explanation and treatment of certain brain diseases, such as memory deletion and literacy barriers, and the further study of cognitive neuroscience.

\section{Acknowledgment}

This work was supported in part by the Scientific Research Project of Beijing Educational Committee (KM201311232007).

\section{References}

[1] J.F. Sun and X.F. Hong, A survey of complex brain networks: Structure, function, computation, and applications, Complex Systems and Complexity Science 4 (2010), 75-78.

[2] M. Jalili and M.G. Knyazeva, Constructing brain functional networks from EEG: Partial vs. un-partial correlations, Journal of Integrative Neuroscience 2 (2011), 58-62.

[3] J.M. Peters and M. Taquet, Brain functional networks in syndromic and non-syndromic autism: A graph theoretical study of EEG connectivity, BMC Medicine 11 (2013), 1-16.

[4] F.F. Zhang and Z.G. Zheng, Complex brain networks: Progress and challenges, Journal of University of Shanghai for Science and Technology 2 (2012), 138-141.

[5] F. Yaghouby, P. Modur, Pradeep Modur and Sridhar Sunderam, Naive scoring of human sleep based on a hidden Markov model of the electroencephalogram, Conference Proceedings of IEEE Engineering in Medical and Biology Society, 2014, pp. 5028-5031.

[6] X.L. Fang and Z.L. Jiang, Analysis of brain function network based EEG, Actaphysica Sinica 12 (2007), 7331-7335.

[7] J.J. Chen and H.F. Li, The analysis and application of brain network genomics research building, Journal of Taiyuan University of Technology 3 (2012), 330-332.

[8] M.G. Kitzbichler and R.N. Henson, Cognitive effort drives workspace configuration of human brain functional networks, Journal of Neuroscience 31(2011), 8259-8270.

[9] C.H. Feng, Pattern recognition research based EEG, Master Dissertation, University of Yanshan, 2011.

[10] L. Wang and X.P. Wu, EEG analysis and classification of four imagine task, Computing Technology and Development 18 (2008), 24-25.

[11] Y.Q. Wang, Construct resting-state brain function network and research on community structure, Master Dissertation, Taiyuan University of Technology, 2012. 